\title{
Mental cultivation (meditation) in Buddhism
}

\author{
Kedar Nath Dwivedi
}

Buddhism started in India in the 6th century BC. It is not a faith, but a way of life that includes maintaining virtuous and moral conduct (Sila), improving the concentration of the mind (Samadhi) and developing insight or wisdom (Panna).

Leading a moral life helps towards better social relationships and mental well-being. There are a minimum of five precepts, which include refraining from killing any sentient beings, lying, stealing, sexual misconduct and use of intoxicants. Maintaining moral conduct is helped by the rituals of 'taking the precepts' and evoking maximum determination and group pressure. If one happens to break the precepts, there is nothing to be gained by feeling guilty; one simply has to re-take them and try again with more determination. The purpose of moral conduct is to achieve good concentration, and good concentration becomes a positive reinforcement for keeping precepts.

In Buddhism, there are many techniques for improving the concentration of mind, or Samadhi in Pali. A common technique called 'Anapan', or meditation on breathing, involves sitting still in a very quiet, dimly lit place, and feeling the touch of the breath on the nostrils while breathing in and out. This has an element of sensory deprivation. Usually, we are not conscious of our muscle tensions, even individuals who are very tense. Such tensions, however, still register on our brains and affect our moods. Thus, sitting still, cutting down thoughts and lowering external sensory stimulation (in a quiet, dimly lit place, eyes closed), will have a relaxation response with calming of the sympathetic nervous system. This is reflected in changes at all three levels: central (alpha waves in the EEG), vegetative (heart rate, breathing, blood pressure) and musculo-skeletal levels (EMG).

Relaxation is an important ingredient in behaviour therapy. Reciprocal inhibition or systematic desensitisation requires both relaxation and exposure to stress. In meditation, too, there is an automatic stirring up of feelings (or stress), even though one is only trying to concentrate on the breath. But, unlike desensitisation, there is no attempt in Anapan meditation to structure these mental contents. If at all, this structuring happens automatically, as if a very powerful computer scans through a vast memory store at lightning speed, selects the most emotionally pressing material that can be tolerated, and processes it through the 'de-magnetising circuits' (Carrington \& Ephron, 1975). In this way, it is rather like free association in psychoanalysis. However, it also differs from psychoanalysis in the sense that it does not involve reporting, analysing or indulging in these thoughts.

The minute trauma of separation, individuation and the narcissistic injuries of the preconceptual, pre-verbal stage in infancy and early life, experienced in silence, culminate to create an unconscious dread of helplessness and passivity, so significant in our day-to-day life. It is difficult to retrieve these pre-verbal traumas through verbalisation in the psychoanalytic situation, but they can be re-visited, re-experienced and mastered non-verbally in the silence of meditation, thus allowing one to reach the parts that may be difficult to gain access to in psychotherapy.

There have been many reviews of the psychophysiological changes, psychological mechanisms and therapeutic effects of different types of meditation (Walsh, 1979). Some of the psychological changes and therapeutic effects include release of tension through catharsis, feeling relaxed and refreshed; availability of affect, getting in touch with feelings, e.g. pleasure, love, sorrow, anger; openness, receptivity, sensitivity; better management of pain by un-coupling of secondary psychological elaboration; reduction in psychosomatic symptoms, e.g. headaches, asthma, insomnia, hypertension; reduction in dependence on alcohol and other substances; getting in touch with conflicting parts of oneself and achieving their synthesis and, thus, releasing a great deal of energy that was blocked away in keeping these parts separate; this release of energy becomes available for creative purposes; and reduction in hostility and anxiety leading to improved performance and learning.

In meditation, one realises that the mind is filled with thoughts that constrict and distort awareness. According to the 'Karmic' principle, 
erroneous viewpoints lead to actions and corrective actions which are mistaken, necessitating further erroneous actions in an infinite regress, turning life into an endless treadmill of suffering (or 'samsara'). Most schools of psychotherapy, especially cognitive psychotherapies, associated with Rational Emotive Therapy (of Ellis), the Assumptive World (of Frank), Personal Constructs (Kelly), and Epistemology (Bateson) aim at exploring and correcting these distorted and erroneous conceptual models of reality, including that of suffering (Ponce, 1982).

In meditation, one begins to see the 'thoughts' for what they are, that is as insubstantial. It is like focusing on an empty sky and watching the clouds come and go, transient, impermanent and changing. The emphasis in meditation is on letting go, non-attachment or renunciation, and not on indulgence or fighting, pushing or pulling. This is in contrast to some schools of psychotherapy where the patient is encouraged to dwell upon these mental contents. But, as there is only one taste throughout the oceans, that of salt, similarly there is only one taste throughout all Buddhist teachings, the idea of renunciation, and of learning that it is safe to 'let go'.

One begins to appreciate that the feeling of ' $\mathrm{I}$ ', 'me' or 'self is just one of the numerous thought forms that flit in and out of consciousness. These thought forms, or the internal processes, seem to have a will of their own; it is futile to control them and, in fact, the so-called ' $\mathrm{I}$ ' or 'self is very impotent in controlling them. A sharply concentrated mind learns to penetrate the illusory processes and to cut through the "delusion of self and permanence". One realises that these are like cartoon films where still pictures are projected so fast that they create the impression of a 'being' with its movements.

We learn in quantum (or particle) physics that mass and energy are the same. At the sub-atomic level, there are just bundles of energy constantly in motion. Nothing is static or unchanging. According to field theory, virtual particles come into being spontaneously out of nothing, and vanish into the same. In experimental physics, while measuring a particle's precise position and its momentum, it is impossible to separate the scientific observer from the observed phenomena. In relativity theory, space and time are interpenetrating, (Capra, 1983; Comfort, 1983). However, our models of psychotherapy are still very Newtonian. Buddhism, on the other hand, provides that experiential basis for such a quantum leap.

Thus, the Buddhist practice of meditation is that of mental cultivation, of penetrating concentration and wisdom (or insight).* The final goal is the attainment of the highest state of disillusionment and enlightenment. This is, however, a very gradual process.

\section{References}

CAPRA, F. (1983) The Tao of Physics. Oxford: Flamingo.

CARRINGTON, P. \& EPHRON, H.S. (1975) Meditation and psychoanalysis. Journal of the American Academy of Psychoanalysis, 3, 43-57.

COMFort, A. (1983) Existential psychiatry and quantum logic. Psychiatry, 46, 383-399.

PONCE, D. (1982) Buddhist constructs and psychotherapy. International Journal of Soctal Psychiatry, 28, 83-90.

WALSH, R. (1979) Meditation research. Journal of Transpersonal Psychology, 11, 161-174.

Kedar Nath Dwivedi, Consultant Child, Adolescent \& Family Psychiatrist, Northampton NN1 2PG

*For example, during the Ten Day Courses held at the International Meditation Centre, Heddington, near Calne, Wiltshire, students start by taking the moral precepts and practising the concentration of mind for the first few days. Once a good degree of concentration is achieved in four to five days, students practise insight, experiencing the changing nature of mind and matter throughout the body for the rest of the course. A continuous effort for several days to sharpen the concentration is analogous to switching on the kettle until the water is boiled. Development of a sharply penetrating concentration allows wisdom to emerge spontaneously through the experience of the rapidly changing mental contents. mood and bodily sensations. 